\title{
Radioactive Seed Implantation and Lobaplatin Chemotherapy Are Safe and Effective in Treating Patients with Advanced Lung Cancer
}

\author{
Jia-Rui Li ${ }^{1}$, Yu Sun ${ }^{2}$, Lin Liu ${ }^{2 *}$
}

\begin{abstract}
Objecive: To investigate the clinical safety and efficacy of CT-guided ${ }^{125}$ iodine (125I) seed implantation combined with percutaneous intra-tumor injection of chemotherapy emulsion of lobaplatin and lipiodol in treating patients with advanced lung cancer. Materials and Methods: Patients with advanced lung cancer and treated with spiral CT-guided ${ }^{125}$ I seed implantation combined with percutaneous intra-tumor injection of chemotherapy emulsion of lobaplatin and lipiodol were recruited. Results: Of the 36 patients, there were 40 nidi in total. The contrast-enhanced CT evaluation was conducted $60 \mathrm{~d}$ after treatment. Response evaluation suggested that 4 patients achieved complete remission (CR), 24 partial remission (PR), 4 stable disease (SD) and 4 progression disease (PD), with a total response rate of $77.8 \%(28 / 36)$. Conclusions: CT-guided ${ }^{125}$ I seed implantation combined with percutaneous intra-tumor injection of chemotherapy emulsion of lobaplatin and lipiodol are safe and effective in treating patients with advanced lung cancer.
\end{abstract}

Keywords: Advanced lung cancer - radioactive seed - chemotherapy emulsion of lobaplatin and lipiodol

Asian Pac J Cancer Prev, 16 (9), 4003-4006

\section{Introduction}

With the development of industrialization and urbanization, the morbidity of lung cancer increases annually (Li et al., 2005). At present, lung cancer, instead of liver cancer, has become the first cause of malignant tumor-related death. Non-small cell lung cancer (NSCLC) and small cell lung cancer (SCLC) are the most common lung cancer in clinic, in which NSCLC accounts for $>85 \%$. As most NSCLC patients $(>85 \%)$ are in advanced stage when diagnosed and have lost the opportunity for radical surgery, the therapeutic efficacy is relatively poor $(\mathrm{Lu}$ et al., 2013; Liu et al., 2013; Yan et al., 2013; Cui et al., 2014; Huang et al., 2014; Ji et al., 2014). In April, 2012, CT-guided ${ }^{125}$ iodine (125I) seed implantation combined with percutaneous intra-tumor injection of chemotherapy emulsion of lobaplatin and lipiodol were applied in our hospital for the treatment of 36 patients with advanced lung cancer, with satisfactory clinical efficacy obtained.

\section{Materials and Methods}

\section{Clinical data}

Of the 36 patients with advanced lung cancer, there were 25 males and 11 females, aged 45 88 years, with the median age of 62.4 years; the maximum diameter of the nidi was $5 \sim 8.6 \mathrm{~mm}$, with median one being $6.2 \mathrm{~mm}$; pathological patterns: 20 patients were with squamous carcinoma, 12 with adenocarcinoma and 4 with SCLC; and clinical stages: 9 in stage III and 27 in stage IV. All patients were pathologically diagnosed as lung cancer and no patient received specialized therapy for tumors before operation.

\section{Preoperative preparation}

The preoperative routine preparations included blood routine, four coagulation tests, electrocardiogram, hepatorenal function, chest X-ray, first-aid instruments and agents. Contrast-enhancement CT was routinely performed to assure the specific locations of lesions, the necrotic areas and the blood flow direction of bloodsupply vessels and adjacent vessels. After the inserting pathways were determined, the seeds were implanted. And then Treatment Planning System (TPS) was applied to distribute the isodoses or I125 empirical formula of Halarism $(\mathrm{mCi}=\mathrm{Da} \times 5)$ was followed. Da was considered to be the average value of the length, width and height $[(\mathrm{L}+\mathrm{W}+\mathrm{H}) \times 5 / 3$; the unit: $\mathrm{cm}]$, and the total activity of ${ }^{125} \mathrm{I}$ seeds and the amounts of the therapeutic seeds was calculated. The dosage of chemotherapy emulsion of lobaplatin and lipiodol was selected according to the distribution of the seeds. The patients were well informed of the operative process before operation and were trained how to hold breath. All informed consent forms were signed by the patients and their families. 
Instruments, equipments and agents

Philips six-row spiral CT machine; radioactive seed source implantation instruments: domestic 18-G radioactive seed source implantation probes and diskrotating implantation gun and China Atomic Energy Research Institute provided IM $67112^{125}$ I seed [NO: SFDA 2001-034]. The activity of a single seed was 23.31 29.97 $\mathrm{MBq}$. Before the seeds left the factory, leakage test was performed and activity was detected. The qualified seeds were packaged and posted to the hospital as A-type. Lobaplatin (Chang'an International Pharmaceutical Co., $L t d$, Hainan, China; specification: $10 \mathrm{mg} /$ piece) was fully mixed with lipiodol to form the emulsion.

\section{Therapeutic methods}

The body position was selected and fixed according to the locations of the lesions. The self-made fence locator was placed on the corresponding surface of the lesions in order to conduct CT scan. CT lines of position were opened and the optimal needling surface was selected and marked. The inserting points, angle, direction and depth were then determined. Sterilization was routinely performed and sterile drapes were set up. After local anesthesia, the patients were told to hold their breath and the puncture probes were inserted till the measuring depth. During the puncture, the CT scan was used to regulate the inserting angle and depth. According to the plan, corresponding amount of radioactive seeds were implanted in designated locations. Based on the surgical requirements, multiple puncture points could be set up on the patient's skin surface. After seed implantation, percutaneous intra-tumor injection of chemotherapy emulsion of lobaplatin and lipiodol was applied for multiple times and points on the locations with residual tumors so as to maximally eliminate the blind space. Postoperative re-examination with CT scan was performed to observe the complications and evaluate the recovery area and the therapeutic satisfactory degree of CT-guided ${ }^{125}$ I seed implantation combined with percutaneous intratumor injection of chemotherapy emulsion of lobaplatin and lipiodol.

\section{Postoperative process}

The amount of seeds and the dosage of chemotherapy emulsion of lobaplatin and lipiodol were examined. After being bandaged, spiral CT thin-layer images (layer thickness: $3 \mathrm{~mm}$ ) were analyzed to observe the presence of the seeds, the metastasis of chemotherapy emulsion lobaplatin and lipiodol and the complications (pneumothorax, hydropneumothorax or pulmonary hemorrhage, etc.). Patients' surveillance: vital signs were closely detected; reptilase $1 \mathrm{Ku}$ was intravenously injected 30 min after operation; antibiotics were routinely applied $3 \mathrm{~d}$ after operation to prevent infection; and continuous low-flow oxygen inhalation was conducted to patients with pulmonary hemorrhage or pneumothorax for $>24 \mathrm{~h}$. For patients with pneumothorax $>30 \%$, chest suction or closed drainage was performed.

\section{Clinical efficacy evaluation}

The efficacy evaluation of thirty-six patients undergoing CT-guided ${ }^{125}$ I seed implantation combined with percutaneous intra-tumor injection of chemotherapy emulsion of lobaplatin and lipiodol mainly depended on the contrast-enhancement spiral CT images. The products of the maximum diameter of the orthogonal tumors detected by spiral CT images before treatment and after the re-examination (60 d after treatment) were compared. The efficacy evaluation referred to the modified Response Evaluation Criteria In Solid Tumors (RECIST) (Yao et al., 2012): Complete remission (CR): the arterial enhancement development of all target nidi disappeared; partial remission (PR): the total diameter of target nidi (arterial enhancement development) shrank $\geq 30 \%$; stable disease (SD): the total diameter of target nidi shrank < that of PR or increased <that of progression disease (PD); PD: total diameter of target nidi (arterial enhancement development) increased $\geq 20 \%$ or new nidi appeared. Total effective rate $=(\mathrm{CR}+\mathrm{PR})$ cases $/$ total cases $\times 100 \%$.

\section{Results}

\section{Postoperative complications}

All patients finished the treatment of CT-guided ${ }^{125} \mathrm{I}$ seed implantation combined with percutaneous intratumor injection of chemotherapy emulsion of lobaplatin and lipiodol, with successful rate being $100 \%$. Antiinfection and analgesia therapies were routinely given after operation. All patients had transient moderate and low heat (temperature $<38.8^{\circ} \mathrm{C}$ ), which recovered to normal level 3 14 d after treatment. Twelve patients, who had slight pneumothorax, were told to stay in bed quietly without giving any special treatment. Only 1 patient had severe pneumothorax (pulmonary compression: about $75 \%$ ) that was completely improved $7 \mathrm{~d}$ after the closed drainage of chest, according to the re-examined chest $\mathrm{X}$-ray images. Twenty patients, who coughed small amount of dark red blood clot or blood streak during and after operation, were given hemostatic therapies. No
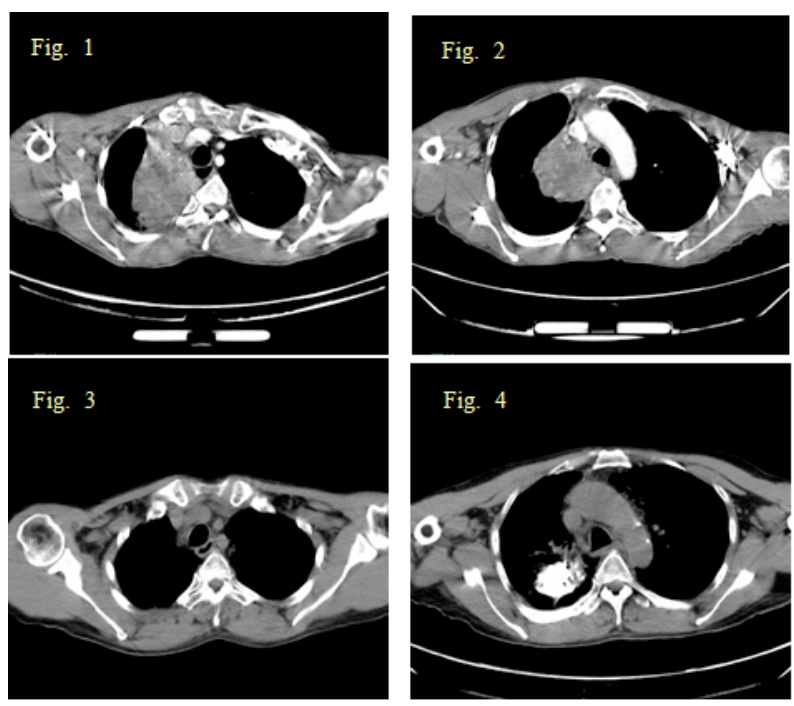

Figures 1 4. Comparison of CT Images before and after Treatment. Male patient, 60 years old. Figures 1 and 2 showed right-upper lung cancer combined with atelectasis according to CT scan before treatment; Figures 3 and 4 showed re-examination results at postoperative $60 \mathrm{~d}$, which suggested that lump shrank markedly and atelectasis recovered to normal 
severe bone marrow function inhibition or gastrointestinal response was observed.

\section{Clinical efficacy}

When hospitalized, all patients had different-degree shortness of breath, dyspnea and chest pain, few of whom also had slight hemoptysis, etc. After treatment, all patients had different-degree improvement in clinical symptoms, especially in chest pain and dyspnea. Re-examined chest CT $60 \mathrm{~d}$ after treatment showed that 4 patients were with CR, 24 with PR, 4 with SD and 4 with PD, with total effective rate being $77.8 \%$ (28/36), as shown in Figures $1 \sim 4$.

\section{Follow up}

The follow-up period was $4 \sim 13$ months, with median follow-up time and rate being 10 months and 100\%, respectively. Moderate- and long-term follow-up results indicated that after 6 months, 28 patients' nidi were stably controlled and 4 died; and after 1 year, only 4 patients' nidi were stably controlled.

\section{Adverse responses}

No severe complication was recorded after treatment. However, there were slight gastrointestinal responses and leukopenia, which were recovered rapidly after the end of treatment. No severe adverse response was reported in all patients.

\section{Discussion}

Radioactive seed implantation in tumor tissues has a long history of $>100$ years, and was firstly applied in treating patients with malignant lung cancers by American Craham in 1933 (Yi et al., 2003). In recent years, with the patients' increasing requirement on the disease therapies and the development of micro-invasive techniques, radioactive seed implantation has achieved rapid progression in the treatment of malignant tumors. ${ }^{125}$ I seeds can cure patients with lung cancer through killing tumor cells by continuously releasing X-ray with radioactive nucleus (Sider et al., 1998; Martinez-Monge et al., 2008) ${ }^{125}$ I seed source is small in size and low in dosage, with half-life period, average photon energy and tissue penetration distance being $59.6 \mathrm{~d}, 28 \mathrm{KeV}$ and 1.7 $\mathrm{cm}$, respectively. It can also release continuously lowdosage $\mathrm{X}$ - and $\gamma$-ray and effectively improve the dosage distribution ratio between locally radioactive tissues and normal tissues. The continuous X-ray irradiation can significantly reduce the regeneration of tumors, while continuous low-dosage X-ray irradiation can inhibit the mitosis of tumor cells and the continuous low-dosage conformal irradiation therapy can evenly irradiate the tumor cells, which conforms to the growth rhythm of tumor cells, thus leading to the maximum killing to the tumor cells due to radiation effect so as to achieve the aim of curing the cancer. Seed therapy belongs to the short-distance radiotherapy field, and the initially developed iodine seed implantation had obtained favorable achievements in the treatment of patients with prostate cancer (PC). Multiple literatures at home and abroad reported the availability of radioactive seed implantation in the treatment of malignant tumors, such as brain cancer, mouth and face cancer, lung cancer, liver cancer, colorectal cancer, PC and pancreatic cancer, etc (Horwitz et al., 1996; Martinez-Monge and Nag, 1999; Barkin et al., 2000; Lee et al., 2003; Liu et al., 2005; Nag et al., 2006; Zhou et al., 2006; Bottomley et al., 2007).

Lobaplatin, as the anti-cancer agent of the thirdgeneration cisplatin, is characterized by wide anti-tumor spectrum, strong anti-tumor activity and low toxic responses, etc (Yang et al., 2009; Huang et al., 2013). In certain range, the tumor-cell-killing function of anticancer agents mainly depends on the agent dosage and application time. After being injected into tumor cells, the chemotherapy emulsion of lobaplatin and lipiodol, which showed different-degree dispersion, could prolong the contact time of lobaplatin with the tumor cells. The local injection of lobaplatin and lipiodol could significantly increase the local agent concentration in tumors while decrease that in non-injection area (Editor-in-chief: Li, 2007). The concentration of locally injected chemotherapy emulsion might become 9 23 folds of that by general chemotherapy in unite time(Zhu et al., 2002). Additionally, the local injection of the emulsion could not only directly kill the tumors, but also prevent the dispersion and metastasis of tumor cells, thus becoming an effective supplement for seed implantation.

All patients finished the treatment of CT-guided ${ }^{125} \mathrm{I}$ seed implantation combined with percutaneous intratumor injection of chemotherapy emulsion of lobaplatin and lipiodol, after which all patients had different-degree improvement in clinical symptoms, especially in chest pain and dyspnea. And the re-examined chest CT $60 \mathrm{~d}$ after treatment showed that 4 patients were with CR, 24 with PR, 4 with SD and 4 with PD, with total effective rate being $77.8 \%$ (28/36). However, the long-term efficacy of the combined treatment should be further observed due to the short term of follow up.

CT-guided ${ }^{125}$ I seed implantation is safe and effective in application, small in trauma, rapid in recovery and slight in postoperative adverse responses, with significant short-term efficacy and short therapeutic duration, so it can be repeatedly applied and has certain clinical efficacy in treating lung cancer patients with various pathological patterns. Therefore, it is believed that CT-guided ${ }^{125} \mathrm{I}$ seed implantation is a relatively safe and effective therapeutic method for middle and advanced lung cancer patients who are poor in surgeries. In this study, no severe adverse response was reported, suggesting that CT-guided ${ }^{125} \mathrm{I}$ seed implantation was an ideal therapeutic method for patients with advanced lung cancer, and it was also concluded that CT-guided ${ }^{125} \mathrm{I}$ seed implantation combined with percutaneous intra-tumor injection of chemotherapy emulsion of lobaplatin and lipiodol could improve the clinical efficacy and prolong the survival time of patients with advanced lung cancer.

\section{References}

Barkin JS, Goldstein JA (2000). Diagnostic and therapeutic approach to pancreatic cancer. Biomed Pharmacother, 54, 
400-9.

Bottomley D, Ash D, Al-Qaisieh B, et al (2007). Side effects of permanent ${ }^{125}$ I prostate seed implants in 667 patients treated in Leeds. Radiother Oncol, 82, 46-9.

Cui L, Liu XX, Jiang Y, et al (2014). Phase II study on dose escalating schedule of paclitaxel concurrent with radiotherapy in treating patients with locally advanced non-small cell lung cancer. Asian Pac J Cancer Prev, 15, 1699-702.

Horwitz EM, Frazier AJ, Martinez AA, et al (1996). Excellent functional outcome in patients with squamous cell carcinoma of the base of tongue treated with external irradiation and interstitial iodine 125 boost. Cancer, 78, 948-57.

Huang XE, Tian GY, Cao J, et al (2014). Pemetrexed as a component of first-, second- and third- line chemotherapy in treating patients with metastatic lung adenocarcinoma. Asian Pac J Cancer Prev, 14, 6663-7.

Huang XE, Wei GL, Huo JG, et al (2013). Intrapleural or intraperitoneal lobaplatin for treatment of patients with malignant pleural effusion or ascites. Asian Pac J Cancer Prev, 14, 2611-4.

Ji ZQ, Huang XE, Wu XY, et al (2014). Safety of Brucea javanica and cantharidin combined with chemotherapy for treatment of NSCLC patients. Asian Pac J Cancer Prev, 15, 8603-5.

Lee W, Daly BD, DiPetrillo TA, et al (2003). Limited resection for non-small cell lung cancer observed local control with implantation of 1125 brachy-therapy seeds. Ann Thorac Surg, 75, 237-42.

Li CJ, Huang JH, Fan WJ, et al (2005). ${ }^{125}$ I seed implant combined with internal iliac arterial infusion chemotherapy in the treatment of recurrence pelvic malignant tumors. $J$ Inter Radiol, 14, 610-2.

Li YH (2007). Illustration on interventional diagnosis and therapeutics in clinical practice (Version 2). Science Press, 7-81.

Liu YC, Zhou SB, Gao F, et al (2013).Chemotherapy and late course three dimensional conformal radiotherapy for treatment of patients with stage III non- small cell lung cancer. Asian Pac J Cancer Prev, 14, 2663-5.

Liu J, Zhang FJ, Wu PH, et al (2005). CT guided radioactive seed ${ }^{125} \mathrm{I}$ implantation in treating hilar hepatic tumor and metastatic lymph nodes. J Inter Radiol, 14, 606-9.

Lu YY, Huang XE, Xu L, et al (2013). Potential predictors of sensitivity to pemetrexed as first-line chemotherapy for patients with advanced non-squamous NSCLCs. Asian Pac J Cancer Prev, 14, 2005-8.

Martinez-Monge R, Nag S et al (1999). Use of combined intraoperative radio-therapy and ${ }^{125} \mathrm{I}$ brachy-therapy in incompletely resected recur-rent colorectal carcinoma. Rev Med Univ Navarra, 43, 11-4.

Martinez-Monge R, Pagola M, Vivas I, et al (2008). CT-guided permanent brachy-therapy for patients with medically inoperable early-stage non-small cell lung cancer NSCLC. Lung Cancer, 61, 209-13.

Nag S, DeHaan M, Scruggs G, et al (2006) . Long-term followup of patients of intra-hepatic malignancies treated with iodine-125 brachy-therapy. Int J Radiat Oncol Biol Phys, 64, 736-44.

Sider L, Mittal BB, Nemced AA Jr, et al (1998). CT guided placement of Iodine-125 seeds for unresectable carcinoma of the lung. J Comput Assist Tomogr, 12, 515-7.

Yang LQ, Qin SK (2009). Progression of lobaplatin as the third generation platinum drug. Chinese Clin Oncol, 14, 1134-9.

Yao XS, Li H (2012). Therapeutic evaluation criterion of inoperable hepatocellular carcinoma: modified RECIST as a more reliable standard. J Inter Radiol, 21, 177-9.

Yan HA, Shen K, Huang XE (2013). Clinical study on mannan peptide combined with TP regimen in treating patients with non-small cell lung cancer. Asian Pac J Cancer Prev, 14, 4801-4.

Yi BY (2003). Clinical nuclide iatreusiology. Beijing: People's Military Medical Press, 169-80.

Zhou K, Wu PH, Zhang FJ, et al (2006). The evaluation of short term effect of CT guided radioactive seed ${ }^{125} \mathrm{I}$ implantation in treating pulmonary metastases from gastrointestinal tumor. J Inter Radiol, 15, 235-7.

Zhu ZD (2002). Pharmacokinetic comparison of chemotherapy fluorouracil through left gastric artery and peripheral vein. Chinese J Gastrointest Surg, 3, 28-30. 